\title{
P-signatures and Noninteractive Anonymous Credentials
}

\author{
Mira Belenkiy $^{1}$, Melissa Chase ${ }^{1}$, Markulf Kohlweiss ${ }^{2}$, and Anna Lysyanskaya ${ }^{1}$ \\ ${ }^{1}$ Brown University \\ \{mira, melissa, anna\}@cs.brown.edu \\ ${ }^{2}$ KU Leuven \\ mkohlwei@esat. kuleuven. be
}

\begin{abstract}
In this paper, we introduce P-signatures. A P-signature scheme consists of a signature scheme, a commitment scheme, and (1) an interactive protocol for obtaining a signature on a committed value; (2) a non-interactive proof system for proving that the contents of a commitment has been signed; (3) a noninteractive proof system for proving that a pair of commitments are commitments to the same value. We give a definition of security for P-signatures and show how they can be realized under appropriate assumptions about groups with a bilinear map. We make extensive use of the powerful suite of non-interactive proof techniques due to Groth and Sahai. Our P-signatures enable, for the first time, the design of a practical non-interactive anonymous credential system whose security does not rely on the random oracle model. In addition, they may serve as a useful building block for other privacy-preserving authentication mechanisms.
\end{abstract}

\section{Introduction}

Anonymous credentials [Cha85, Dam90, Bra99, LRSW99, CL01, CL02, CL04] let A1ice prove to Bob that Carol has given her a certificate. Anonymity means that Bob and Carol cannot link Alice's request for a certificate to Alice's proof that she possesses a certificate. In addition, if Alice proves possession of a certificate multiple times, these proofs cannot be linked to each other. Anonymous credentials are an example of a privacy-preserving authentication mechanism, which is an important theme in modern cryptographic research. Other examples are electronic cash [CFN90, CP93, Bra93. CHL05] and group signatures [CvH91, CS97, ACJT00, BBS04, BW06, BW07]. In a series of papers, Camenisch and Lysyanskaya [CL01, CL02, CL04] identified a key building block commonly called "a CL-signature" that is frequently used in these constructions. A CL-signature is a signature scheme with a pair of useful protocols.

The first protocol, called Issue, lets a user obtain a signature on a committed message without revealing the message. The user wishes to obtain a signature on a value $x$ from a signer with public key $p k$. The user forms a commitment comm to value $x$ and gives comm to the signer. After running the protocol, the user obtains a signature on $x$, and the signer learns no information about $x$ other than the fact that he has signed the value that the user has committed to.

The second protocol, called Prove, is a zero-knowledge proof of knowledge of a signature on a committed value. The prover has a message-signature pair $\left(x, \sigma_{p k}(x)\right)$.

R. Canetti (Ed.): TCC 2008, LNCS 4948, pp. 356-374, 2008.

(C) International Association for Cryptologic Research 2008 
The prover has obtained it by either running the Issue protocol, or by querying the signer on $x$. The prover also has a commitment comm to $x$. The verifier only knows comm. The prover proves in zero-knowledge that he knows a pair $(x, \sigma)$ and a value open such that $\operatorname{Verify} \operatorname{Sig}(p k, x, \sigma)=$ accept and $\operatorname{comm}=\operatorname{Commit}(x$, open $)$.

It is clear that using general secure two-party computation [Yao86] and zero-knowledge proofs of knowledge of a witness for any NP statement [GMW86], we can construct the Issue and Prove protocols from any signature scheme and commitment scheme. Camenisch and Lysyanskaya's contribution was to construct specially designed signature schemes that, combined with Pedersen [Ped92] and Fujisaki-Okamoto [FO98] commitments, allowed them to construct Issue and Prove protocols that are efficient enough for use in practice. In turn, CL-signatures have been implemented and standardized [CVH02, [BCC04]. They have also been used as a building block in many other constructions [JS04, BCL04, CHL05, DDP06, $\left.\mathrm{CHK}^{+} 06, \mathrm{TS} 06\right]$ ].

A shortcoming of the CL signature schemes is that the Prove protocol is interactive. Rounds of interaction are a valuable resource. In certain contexts, proofs need to be verified by third parties who are not present during the interaction. For example, in offline e-cash, a merchant accepts an e-coin from a buyer and later deposits the e-coin to the bank. The bank must be able to verify that the e-coin is valid.

There are two known techniques for making the CL Prove protocols non-interactive. We can use the Fiat-Shamir heuristic [FS87], which requires the random-oracle model. A series of papers [CGH04, DNRS03, GK03] show that proofs of security in the randomoracle model do not imply security. The other option is to use general techniques: [BFM88, DSMP88, BDMP91] show how any statement in NP can be proven in noninteractive zero-knowledge. This option is prohibitively expensive.

We give the first practical non-interactive zero-knowledge proof of knowledge of a signature on a committed message. We have two constructions using two different practical siganture schemes and a special class of commitments due to Groth and Sahai [GS07]. Our constructions are secure in the common reference string model.

Due to the fact that these protocols are so useful for a variety of applications, it is important to give a careful treatment of the security guarantees they should provide. In this paper, we introduce the concept of P-signatures — signatures with efficient Protocols, and give a definition of security. The main difference between P-signatures and CLsignatures is that $\mathrm{P}$-signatures have non-interactive proof protocols. (Our definition can be extended to encompass CL signatures as well.)

Our CONTRIBUtions. Our main contribution is the formal definition of a P-signature scheme and two efficient constructions.

Anonymous credentials are an immediate consequence of P-signatures (and of CLsignatures [Lys02]). Let us explain why (see full paper for an in-depth treatment). Suppose there is a public-key infrastructure that lets each user register a public key. Alice registers unlinkable pseudonyms $A_{B}$ and $A_{C}$ with Bob and Carol. $A_{B}$ and $A_{C}$ are commitments to her secret key, and so they are unlinkable by the security properties of the commitment scheme. Suppose Alice wishes to obtain a certificate from Carol and show it to Bob. Alice goes to Carol and identifies herself as the owner of pseudonym $A_{C}$. They run the P-signature Issue protocol as a result of which Alice gets 
Carol's signature on her secret key. Now Alice uses the P-signature Prove protocol to construct a non-interactive proof that she has Carol's signature on the opening of $A_{B}$.

Our techniques may be of independent interest. Typically, a proof of knowledge $\pi$ of a witness $x$ to a statement $s$ implies that there exists an efficient algorithm that can extract a value $x^{\prime}$ from $\pi$ such that $x^{\prime}$ satisfies the statement $s$. Our work uses GrothSahai non-interactive proofs of knowledge [GS07] from which we can only extract $f(x)$ where $f$ is a one-way function. We formalize the notion of an $f$-extractable proof of knowledge and develop useful notation for describing $f$-extractable proofs that committed values have certain properties. Our notation has helped us understand how to work with the GS proof system and it may encourage others to use the wealth of this powerful building block.

TECHNICAL ROADMAP. We use Groth and Sahai's $f$-extractable non-interactive proofs of knowledge [GS07] to build P-signatures. Groth and Sahai give three instantiations for their proof system, using SXDH, DLIN, and SDA assumptions. We can use either of the first two instantiations. The SDA-based instantiation does not give us the necessary extraction properties.

Another issue we confront is that Groth-Sahai proofs are $f$-extractable and not fully extractable. Suppose we construct a proof whose witness $x$ contains $a \in Z_{p}$ and the opening of a commitment to $a$. For this commitment, we can only extract $b^{a} \in f(x)$ from the proof, for some base $b$. Note that the proof can be about multiple committed values. Thus, if we construct a proof of knowledge of $(m, \sigma)$ where $m \in Z_{p}$ and $\operatorname{Verify} \operatorname{Sig}(p k, m, \sigma)=$ accept, we can only extract some function $F(m)$ from the proof. However, even if it is impossible to forge $(m, \sigma)$ pairs, it might be possible to forge $(F(m), \sigma)$ pairs. Therefore, for our proof system to be meaningful, we need to define $F$-unforgeable signature schemes, i.e. schemes where it is impossible for an adversary to compute a $(F(m), \sigma)$ pair on his own.

Our first construction uses the Weak Boneh-Boyen (WBB) signature scheme [BB04]. Using a rather strong assumption, we prove that WBB is $F$-unforgeable and our Psignature construction is secure. Our second construction uses a better assumption (because it is falsfiable [Nao03]) and Our construction is based on the Full Boneh-Boyen signature scheme [BB04]. We had to modify the Boneh-Boyen construction, however, because the GS proof system would not allow the knowledge extraction of the entire signature. Our first construction is much simpler, but, as it's security relies on an interactive and thus much stronger assumption, we have decided to focus here on our second construction. For details on the first construction, see the full version.

Organization. Sections 2 and 3 define P-signatures and introduce complexity assumptions. Section 4 explains non-interactive proofs of knowledge, introduces our new notation, and reviews GS proofs. Finally, Section 5 contains our second construction.

\section{Definition of a Secure P-signature Scheme}

We say that a function $\nu: \mathbb{Z} \rightarrow \mathbb{R}$ is negligible if for all integers $c$ there exists an integer $K$ such that $\forall k>K,|\nu(k)|<1 / k^{c}$. We use the standard GMR [GMR88] notation to describe probability spaces. 
Here we introduce P-signatures a primitive which lets a user (1) obtain a signature on a committed message without revealing the message, (2) construct a non-interactive zero-knowledge proof of knowledge of $(F(m), \sigma)$ such that $\operatorname{Verify} \operatorname{Sig}(p k, m, \sigma)=$ accept and $m$ is committed to in a commitment comm, and (3) a non-interactive method for proving that a pair of commitments are to the same value. In this section, we give the first formal definition of a non-interactive P-signature scheme. We begin by reviewing digital signatures and introducing the concept of $F$-unforgeability.

\subsection{Digital Signatures}

A signature scheme consists of four algorithms: SigSetup, Keygen, Sign, and VerifySig. $\operatorname{SigSetup}\left(1^{k}\right)$ generates public parameters params $_{S i g}$. Keygen $\left(\right.$ params $\left._{\text {Sig }}\right)$ generates signing keys $(p k, s k)$. Sign $\left(\right.$ params $\left._{\text {Sig }}, s k, m\right)$ computes a signature $\sigma$ on $m$. VerifySig (params $\left._{\text {Sig }}, p k, m, \sigma\right)$ outputs accept if $\sigma$ is a valid signature on $m$, reject if not.

The standard definition of a secure signature scheme [GMR88] states that no adversary can output $(m, \sigma)$, where $\sigma$ is a signature on $m$, without first previously obtaining a signature on $m$. This is insufficient for our purposes. Our P-Signature constructions prove that we know some value $y=F(m)$ (for an efficiently computable bijection $F$ ) and a signature $\sigma$ such that VerifySig $\left(\right.$ params $\left._{\text {Sig }}, p k, m, \sigma\right)=$ accept. However, even if an adversary cannot output $(m, \sigma)$ without first obtaining a signature on $m$, he might be able to output $(F(m), \sigma)$. Therefore, we introduce the notion of $F$-Unforgeability:

Definition 1 ( $F$-Secure Signature Scheme). We say that a signature scheme is $F$ secure (against adaptive chosen message attacks) if it is Correct and F-Unforgeable.

Correct. VerifySig always accepts a signature obtained using the Sign algorithm.

$F$-Unforgeable. Let $F$ be an efficiently computable bijection. No adversary should be able to output $(F(m), \sigma)$ unless he has previously obtained a signature on $m$. Formally, for every PPTM adversary $\mathcal{A}$, there exists a negligible function $\nu$ such that

$$
\begin{aligned}
& \operatorname{Pr}\left[\text { params }_{\text {Sig }} \leftarrow \operatorname{SigSetup}\left(1^{k}\right) ;(p k, \text { sk }) \leftarrow \operatorname{Keygen}_{\left(\text {params }_{\text {Sig }}\right)}\right) \\
& \left(Q_{\text {Sign }}, y, \sigma\right) \leftarrow \mathcal{A}\left(\text { params }_{\text {Sig }}, p k\right)^{\mathcal{O}_{\text {Sign }}\left(\text { params }_{\text {Sig }}, \text { sk }, \cdot\right)}: \\
& \left.\quad \operatorname{VerifySig}\left(\text { params }_{\text {Sig }}, p k, F^{-1}(y), \sigma\right)=1 \wedge y \notin F\left(Q_{\text {Sign }}\right)\right]<\nu(k) .
\end{aligned}
$$

$\mathcal{O}_{\text {Sign }}\left(\right.$ params $\left._{\text {Sig }}, s k, m\right)$ records $m$-queries on $Q_{\text {Sign }}$ and returns $\operatorname{Sign}\left(\right.$ params $_{\text {Sig }}$, $s k, m) . F\left(Q_{\mathrm{Sign}}\right)$ evaluates $F$ on all values on $Q_{\mathrm{Sign}}$.

Lemma 1. F-unforgeable signatures are secure in the standard [GMR88] sense.

Proof sketch. Suppose an adversary can compute a forgery $(m, \sigma)$. Now the reduction can use it to compute $(F(m), \sigma)$.

\subsection{Commitment Schemes}

Recall the standard definition of a non-interactive commitment scheme. It consists of algorithms ComSetup, Commit. ComSetup $\left(1^{k}\right)$ outputs public parameters params ${ }_{\text {Com }}$ 
for the commitment scheme. Commit $\left(\right.$ params $_{\text {Com }}, x$, open $)$ is a deterministic function that outputs comm, a commitment to $x$ using auxiliary information open. We need commitment schemes that are perfectly binding and strongly computationally hiding:

Perfectly Binding. For every bitstring comm, there exists at most one value $x$ such that there exists opening information open so that comm $=$ Commit (params, $x$, open $)$. We also require that it be easy to identify the bitstrings $c o m m$ for which there exists such an $x$.

Strongly Computationally Hiding. There exists an alternate setup HidingSetup $\left(1^{k}\right)$ that outputs parameters (computationally indistinguishable from the output of ComSetup $\left(1^{k}\right)$ ) so that the commitments become information-theoretically hiding.

\subsection{Non-interactive P-signatures}

A non-interactive P-signature scheme extends a signature scheme (Setup, Keygen, Sign, VerifySig) and a non-interactive commitment scheme (Setup, Commit). It consists of the following algorithms (Setup, Keygen, Sign, VerifySig, Commit, ObtainSig, IssueSig, Prove, VerifyProof, EqCommProve, VerEqComm).

Setup $\left(1^{k}\right)$. Outputs public parameters params. These parameters include parameters for the signature scheme and the commitment scheme.

ObtainSig (params, pk, $m$, comm, open $) \leftrightarrow$ IssueSig(params, sk, comm). These two interactive algorithms execute a signature issuing protocol between a user and the issuer. The user takes as input (params, pk, $m$, comm, open) such that the value comm $=$ Commit (params, $m$, open $)$ and gets a signature $\sigma$ as output. If this signature does not verify, the user sends "reject" to the issuer. The issuer gets (params, sk, comm) as input and gets nothing as output.

Prove $($ params $, p k, m, \sigma)$. Outputs the values $(c o m m, \pi$, open $)$, such that comm = Commit (params, $m$, open) and $\pi$ is a proof of knowledge of a signature $\sigma$ on $m$.

VerifyProof (params, $p k, c o m m, \pi)$. Takes as input a commitment to a message $m$ and a proof $\pi$ that the message has been signed by owner of public key $p k$. Outputs accept if $\pi$ is a valid proof of knowledge of $F(m)$ and a signature on $m$, and outputs reject otherwise.

EqCommProve (params, $m$, open, open' ${ }^{\prime}$. Takes as input a message and two commitment opening values. It outputs a proof $\pi$ that $\operatorname{comm}=\operatorname{Commit}(m$,open $)$ is a commitment to the same value as comm ${ }^{\prime}=\operatorname{Commit}\left(m, o p e n^{\prime}\right)$. This proof is used to bind the commitment of a P-signature proof to a more permanent commitment.

VerEqComm (params, comm, comm $\left.{ }^{\prime}, \pi\right)$. Takes as input two commitments and a proof and accepts if $\pi$ is a proof that comm, comm' are commitments to the same value.

Definition 2 (Secure P-Signature Scheme). Let $F$ be a efficiently computable bijection (possibly parameterized by public parameters). A P-signature scheme is secure if (Setup, Keygen, Sign, VerifySig) form an F-unforgeable signature scheme, if (Setup, Commit) is a perfectly binding, strongly computationally hiding commitment scheme, if (Setup, EqCommProve, VerEqComm) is a non-interactive proof system, and if the Signer privacy, User privacy, Correctness, Unforgeability, and Zero-knowledge properties hold: 
Correctness. An honest user who obtains a P-signature from an honest issuer will be able to prove to an honest verifier that he has a valid signature.

Signer privacy. No PPTM adversary can tell if it is running IssueSig with an honest issuer or with a simulator who merely has access to a signing oracle. Formally, there exists a simulator Simlssue such that for all PPTM adversaries $\left(\mathcal{A}_{1}, \mathcal{A}_{2}\right)$, there exists a negligible function $\nu$ so that:

$$
\begin{aligned}
& \mid \operatorname{Pr}\left[\text { params } \leftarrow \operatorname{Setup}\left(1^{k}\right) ;(\text { sk }, \text { pk }) \leftarrow \text { Keygen }(\text { params }) ;\right. \\
& \quad(\text { m, open }, \text { state }) \leftarrow \mathcal{A}_{1}(\text { params }, \text { sk }) ; \\
& \quad \text { comm } \leftarrow \text { Commit }(\text { params }, m, \text { open }) ; \\
& \left.\quad b \leftarrow \mathcal{A}_{2}(\text { state }) \leftrightarrow \operatorname{IssueSig}(\text { params }, \text { sk }, \text { comm }): b=1\right] \\
& -\operatorname{Pr}\left[\text { params } \leftarrow \text { Setup }\left(1^{k}\right) ;(\text { sk }, \text { pk }) \leftarrow \operatorname{Keygen}(\text { params }) ;\right. \\
& \quad(m, \text { open }, \text { state }) \leftarrow \mathcal{A}_{1}(\text { params }, \text { sk }) ; \\
& \quad \text { comm } \leftarrow \text { Commit }(\text { params }, m, \text { open }) ; \sigma \leftarrow \operatorname{Sign}(\text { params }, \text { sk, } m) ; \\
& \left.\quad b \leftarrow \mathcal{A}_{2}(\text { state }) \leftrightarrow \operatorname{Simlssue}(\text { params }, \text { comm }, \sigma): b=1\right] \mid<\nu(k)
\end{aligned}
$$

Note that we ensure that IssueSig and Simlssue gets an honest commitment to whatever $m$, open the adversary chooses.

Since the goal of signer privacy is to prevent the adversary from learning anything except a signature on the opening of the commitment, this is sufficient for our purposes. Note that our SimIssue will be allowed to rewind $\mathcal{A}$. to Also, we have defined Signer Privacy in terms of a single interaction between the adversary and the issuer. A simple hybrid argument can be used to show that this definition implies privacy over many sequential instances of the issue protocol.

User privacy. No PPTM adversary $\left(\mathcal{A}_{1}, \mathcal{A}_{2}\right)$ can tell if it is running ObtainSig with an honest user or with a simulator. Formally, there exists a simulator $\operatorname{Sim}=\operatorname{SimObtain}$ such that for all PPTM adversaries $\mathcal{A}_{1}, \mathcal{A}_{2}$, there exists negligible function $\nu$ so that:

$$
\begin{aligned}
& \mid \operatorname{Pr}\left[\text { params } \leftarrow \operatorname{Setup}\left(1^{k}\right) ;(\text { p }, m, \text { open }, \text { state }) \leftarrow \mathcal{A}_{1}(\text { params }) ;\right. \\
& \quad \text { comm }=\operatorname{Commit}(\text { params }, m, \text { open }) ; \\
& \left.\quad b \leftarrow \mathcal{A}_{2}(\text { state }) \leftrightarrow \text { ObtainSig }(\text { params }, \text { pk }, m, \text { comm }, \text { open }): b=1\right] \\
& -\operatorname{Pr}\left[(\text { params }, \text { sim }) \leftarrow \operatorname{Setup}\left(1^{k}\right) ;(\text { pk }, m, \text { open }, \text { state }) \leftarrow \mathcal{A}_{1}(\text { params }) ;\right. \\
& \quad \text { comm }=\text { Commit }(\text { params }, m, \text { open }) ; \\
& \left.\quad b \leftarrow \mathcal{A}_{2}(\text { state }) \leftrightarrow \operatorname{SimObtain}(\text { params }, p k, \text { comm }): b=1\right] \mid<\nu(k)
\end{aligned}
$$

Here again SimObtain is allowed to rewind the adversary.

Note that we require that only the user's input $m$ is hidden from the issuer, but not necessarily the user's output $\sigma$. The reason that this is sufficient is that in actual applications (for example, in anonymous credentials), a user would never show $\sigma$ in the clear; instead, he would just prove that he knows $\sigma$. An alternative, stronger way to define signer privacy and user privacy together, would be to require that the pair of algorithms ObtainSig and IssueSig carry out a secure two-party computation. This alternative definition would ensure that $\sigma$ is hidden from the issuer as well. However, as explained above, this feature is not necessary for our application, so we preferred to give a special definition which captures the minimum properties required. 
Unforgeability. We require that no PPTM adversary can create a proof for any message $m$ for which he has not previously obtained a signature or proof from the oracle.

A P-signature scheme is unforgeable if an extractor (ExtractSetup, Extract) and a bijection $F$ exist such that (1) the output of ExtractSetup $\left(1^{k}\right)$ is indistinguishable from the output of $\operatorname{Setup}\left(1^{k}\right)$, and (2) no PPTM adversary can output a proof $\pi$ that VerifyProof accepts, but from which we extract $F(m), \sigma$ such that either (a) $\sigma$ is not valid signature on $m$, or (b) comm is not a commitment to $m$ or (c) the adversary has never previously queried the signing oracle on $m$. Formally, for all PPTM adversaries $\mathcal{A}$, there exists a negligible function $\nu$ such that:

$$
\begin{aligned}
& \operatorname{Pr}\left[\text { params }_{0} \leftarrow \operatorname{Setup}\left(1^{k}\right) ;\left(\text { params }_{1}, t d\right) \leftarrow \text { ExtractSetup }\left(1^{k}\right): b \leftarrow\{0,1\}:\right. \\
& \left.\mathcal{A}\left(\text { params }_{b}\right)=b\right]<1 / 2+\nu(k) \text {, and } \\
& \operatorname{Pr}\left[(\text { params }, t d) \leftarrow \text { ExtractSetup }\left(1^{k}\right) ;(p k, s k) \leftarrow \text { Keygen }(\text { params }) ;\right. \\
& \left(Q_{\mathrm{Sign}}, \mathrm{comm}, \pi\right) \leftarrow \mathcal{A}(\text { params }, p k)^{\mathcal{O}_{\mathrm{Sign}}(\text { params }, s k, \cdot)} ; \\
& (y, \sigma) \leftarrow \operatorname{Extract}(\text { params }, t d, \pi, \text { comm }): \\
& \text { VerifyProof (params, } p k, \text { comm }, \pi)=\text { accept } \\
& \wedge\left(\text { VerifySig }\left(\text { params }, p k, F^{-1}(y), \sigma\right)=\right.\text { reject } \\
& \vee\left(\forall \text { open }, \text { comm } \neq \text { Commit }\left(\text { params }, F^{-1}(y) \text {, open }\right)\right) \\
& \left.\left.\vee\left(\operatorname{VerifySig}\left(\text { params }, p k, F^{-1}(y), \sigma\right)=\text { accept } \wedge y \notin F\left(Q_{\text {Sign }}\right)\right)\right)\right]<\nu(k) \text {. }
\end{aligned}
$$

Oracle $\mathcal{O}_{\text {Sign }}($ params, sk, $m$ ) runs the function $\operatorname{Sign}($ params, $s k, m)$ and returns the resulting signature $\sigma$ to the adversary. It records the queried message on query tape $Q_{\text {Sign }}$. By $F\left(Q_{\text {Sign }}\right)$ we mean $F$ applied to every message in $Q_{\text {Sign }}$.

Zero-knowledge. There exists a simulator $\operatorname{Sim}=$ (SimSetup, SimProve, SimEqComm), such that for all PPTM adversaries $\mathcal{A}_{1}, \mathcal{A}_{2}$, there exists a negligible function $\nu$ such that under parameters output by SimSetup, Commit is perfectly hiding and (1) the parameters output by SimSetup are indistinguishable from those output by Setup, but SimSetup also outputs a special auxiliary string sim; (2) when params are generated by SimSetup, the output of SimProve (params, sim, pk) is indistinguishable from that of Prove (params, $p k, m, \sigma)$ for all $(p k, m, \sigma)$ where $\sigma \in \sigma_{p k}(m)$; and (3) when params are generated by SimSetup, the output of

SimEqComm (params, sim, comm, comm ${ }^{\prime}$ ) is indistinguishable from that of EqCommProve (params, $m$, open, open $\left.{ }^{\prime}\right)$ for all ( $m$, open, open') where comm $=$ Commit $($ params, $m$, open $)$ and comm ${ }^{\prime}=$ Commit $\left(\right.$ params, $m$,open $\left.{ }^{\prime}\right)$. In GMR notation, this is formally defined as follows:

$$
\begin{aligned}
& \mid \operatorname{Pr}\left[\text { params } \leftarrow \operatorname{Setup}\left(1^{k}\right) ; b \leftarrow \mathcal{A}(\text { params }): b=1\right] \\
& \quad-\operatorname{Pr}\left[(\text { params }, \text { sim }) \leftarrow \operatorname{SimSetup}\left(1^{k}\right) ; b \leftarrow \mathcal{A}(\text { params }): b=1\right] \mid<\nu(k), \text { and } \\
& \mid \operatorname{Pr}\left[(\text { params }, \text { sim }) \leftarrow \operatorname{SimSetup~}\left(1^{k}\right) ;(\text { pk }, m, \sigma, \text { state }) \leftarrow \mathcal{A}_{1}(\text { params }, \text { sim }) ;\right. \\
& \left.\quad(\text { comm }, \pi, \text { open }) \leftarrow \operatorname{Prove}(\text { params }, p k, m, \sigma) ; b \leftarrow \mathcal{A}_{2}(\text { state }, \text { comm }, \pi): b=1\right] \\
& -\operatorname{Pr}\left[(\text { params }, \text { sim }) \leftarrow \operatorname{SimSetup}\left(1^{k}\right) ;(p k, m, \sigma, \text { state }) \leftarrow \mathcal{A}_{1}(\text { params }, \text { sim }) ;\right. \\
& \quad(\text { comm }, \pi) \leftarrow \operatorname{SimProve}(\text { params }, \text { sim }, p k) ; b \leftarrow \mathcal{A}_{2}(\text { state }, \text { comm }, \pi) \\
& \quad: b=1] \mid<\nu(k), \text { and }
\end{aligned}
$$




$$
\begin{aligned}
& \mid \operatorname{Pr}\left[(\text { params }, \text { sim }) \leftarrow \operatorname{SimSetup}\left(1^{k}\right) ;\left(\text { m, open }, \text { open }^{\prime}\right) \leftarrow \mathcal{A}_{1}(\text { params }, \text { sim }) ;\right. \\
& \left.\pi \leftarrow \text { EqCommProve }\left(\text { params, } m, \text { open, open }{ }^{\prime}\right) ; b \leftarrow \mathcal{A}_{2}(\text { state }, \pi): b=1\right] \\
& -\operatorname{Pr}\left[(\text { params }, \text { sim }) \leftarrow \operatorname{SimSetup}\left(1^{k}\right) ;\left(\text { m, open }, \text { open }{ }^{\prime}\right) \leftarrow \mathcal{A}_{1}(\text { params }, \text { sim }) ;\right. \\
& \pi \leftarrow \operatorname{SimEqComm}(\text { params, sim, Commit(params, } m \text {, open }) \text {, } \\
& \text { Commit (params, } \left.\left.m \text {, open }{ }^{\prime}\right)\right) \text {; } \\
& \left.b \leftarrow \mathcal{A}_{2}(\text { state }, \pi): b=1\right] \mid<\nu(k) .
\end{aligned}
$$

\section{Preliminaries}

Let $G_{1}, G_{2}$, and $G_{T}$ be groups. A function $e: G_{1} \times G_{2} \rightarrow G_{T}$ is called a cryptographic bilinear map if it has the following properties: Bilinear. $\forall a \in G_{1}, \forall b \in G_{2}, \forall x, y \in \mathbb{Z}$ the following equation holds: $e\left(a^{x}, b^{y}\right)=e(a, b)^{x y}$. Non-Degenerate. If $a$ and $b$ are generators of their respective groups, then $e(a, b)$ generates $G_{T}$. Let BilinearSetup $\left(1^{k}\right)$ be an algorithm that generates the groups $G_{1}, G_{2}$ and $G_{T}$, together with algorithms for sampling from these groups, and the algorithm for computing the function $e$.

The function BilinearSetup $\left(1^{k}\right)$ outputs $\operatorname{params}_{B M}=\left(p, G_{1}, G_{2}, G_{T}, e, g, h\right)$, where $p$ is a prime (of length $k$ ), $G_{1}, G_{2}, G_{T}$ are groups of order $p, g$ is a generator of $G_{1}, h$ is a generator of $G_{2}$, and $e: G_{1} \times G_{2} \rightarrow G_{T}$ is a bilinear map.

We introduce a new assumption which we call TDH and review the HSDH assumption introduced by Boyen and Waters [BW07]. Groth-Sahai proofs use either the DLIN [BBS04] or SXDH [Sco02] assumption. For formal definitions, see the full version.

Definition 3 (Triple DH (TDH)). On input $g, g^{x}, g^{y}, h, h^{x},\left\{c_{i}, g^{1 /\left(x+c_{i}\right)}\right\}_{i=1 \ldots q}$, it is computationally infeasible to output a tuple $\left(h^{\mu x}, g^{\mu y}, g^{\mu x y}\right)$ for $\mu \neq 0$.

Definition 4 (Hidden SDH [BW07]). On input $g, g^{x}, u \in G_{1}, h, h^{x} \in G_{2}$ and $\left\{g^{1 /\left(x+c_{\ell}\right)}, h^{c_{\ell}}, u^{c_{\ell}}\right\}_{\ell=1 \ldots q}$, it is computationally infeasible to output a new tuple $\left(g^{1 /(x+c)}, h^{c}, u^{c}\right)$.

Definition 5 (Decisional Linear Assumption (DLIN)). On input $u, v, w, u^{r}, v^{s} \leftarrow$ $G_{1}$ it is computationally infeasible to distinguish $z_{0} \leftarrow w^{r+s}$ from $z_{1} \leftarrow G_{1}$. The assumption is analogously defined for $G_{2}$.

Definition 6 (Symmetric External Diffie-Hellman Assumption (SXDH)). SXDH states that the Decisional Diffie Hellman problem is hard in both $G_{1}$ and $G_{2}$. This precludes efficient isomorphisms between these two groups.

\section{Non-interactive Proofs of Knowledge}

Our P-signature constructions use the Groth and Sahai [GS07] non-interactive proof of knowledge (NIPK) system. De Santis et al. [DDP00] give the standard definition of NIPK systems. Their definition does not fully cover the Groth and Sahai proof system. In this section, we review the standard notion of NIPK. Then we give a useful generalization, which we call an $f$-extractable NIPK, where the extractor only extracts a 
function of the witness. We develop useful notation for expressing $f$-extractable NIPK systems, and explain how this notation applies to the Groth-Sahai construction. We then review Groth-Sahai commitments and pairing product equation proofs. Finally, we show how they can be used to prove statments about committed exponents, as this will be necessary later for our constructions.

\subsection{Proofs of Knowledge: Notation and Definitions}

In this subsection, we review the definition of NIPK, introduce the notion of $f$-extractability, and develop some useful notation. We review the De Santis et al. [DDP00] definition of NIPK. Let $L=\left\{s: \exists x\right.$ s.t. $M_{L}(s, x)=$ accept $\}$ be a language in NP and $M_{L}$ a polynomial-time Turing Machine that verifies that $x$ is a valid witness for the statement $s \in L$. A NIPK system consists of three algorithms: (1) PKSetup $\left(1^{k}\right)$ sets up the common parameters params $_{P K}$; (2) PKProve $\left(\right.$ params $\left._{P K}, s, x\right)$ computes a proof $\pi$ of the statement $s \in L$ using witness $x$; (3) PKVerify $\left(\operatorname{params}_{P K}, s, \pi\right)$ verifies correctness of $\pi$. The system must be complete and extractable. Completeness means that for all values of params $_{P K}$ and for all $s, x$ such that $M_{L}(s, x)=$ accept, a proof $\pi$ generated by PKProve $\left(\operatorname{param}_{P K}, s, x\right)$ must be accepted by PKVerify $\left(\operatorname{params}_{P K}, s, \pi\right)$. Extractability means that there exists a polynomial-time extractor (PKExtractSetup, PKExtract). PKExtractSetup $\left(1^{k}\right)$ outputs $\left(t d\right.$, params $\left._{P K}\right)$ where params $_{P K}$ is distributed identically to the output of PKSetup $\left(1^{k}\right)$. For all PPT adversaries $\mathcal{A}$, the probability that $\mathcal{A}\left(1^{k}\right.$, params $\left._{P K}\right)$ outputs $(s, \pi)$ such that PKVerify $\left(\operatorname{params}_{P K}, s, \pi\right)=$ accept and PKExtract $(t d, s, \pi)$ fails to extract a witness $x$ such that $M_{L}(s, x)=$ accept is negligible in $k$. We have perfect extractability if this probability is 0 .

We first generalize the notion of NIPK for a language $L$ to languages parameterized by $\operatorname{prams}_{P K}$ - we allow the Turing machine $M_{L}$ to receive $\operatorname{params}_{P K}$ as a separate input. Next, we generalize extractability to $f$-extractability. We say that a NIPK system is $f$-extractable if PKExtract outputs $y$, such that there $\exists x: M_{L}\left(\right.$ params $\left._{P K}, s, x\right)=$ accept $\wedge y=f\left(\right.$ params $\left._{P K}, x\right)$. If $f\left(\right.$ params $\left._{P K}, \cdot\right)$ is the identity function, we get the usual notion of extractability. We denote an $f$-extractable proof $\pi$ obtained by running PKProve $\left(\operatorname{params}_{P K}, s, x\right)$ as

$$
\pi \leftarrow \operatorname{NIPK}\left\{\text { params }_{P K}, s, f\left(\text { params }_{P K}, x\right): M_{L}\left(\text { params }_{P K}, s, x\right)=\text { accept }\right\} .
$$

We omit the params $_{P K}$ where they are obvious. In our applications, $s$ is a conditional statement about the witness $x$, so $M_{L}(s, x)=$ accept if Condition $(x)=\operatorname{accept}$. Thus the statement $\pi \leftarrow \operatorname{NIPK}\{f(x)$ : Condition $(x)\}$ is well defined. Suppose $s$ includes a list of commitments $c_{n}=\operatorname{Commit}\left(x_{n}\right.$, open $\left._{n}\right)$. The witness is $x=\left(x_{1}, \ldots, x_{N}\right.$, open $_{1}, \ldots$, open $\left.{ }_{N}\right)$, however, we typically can only extract $x_{1}, \ldots, x_{N}$. We write

$$
\begin{aligned}
\pi \leftarrow \operatorname{NIPK}\left\{\left(x_{1}, \ldots, x_{n}\right)\right. & : \text { Condition }(x) \\
& \left.\wedge \forall \ell \exists \text { open }_{\ell}: c_{\ell}=\text { Commit }\left(\text { params }_{\text {Com }}, x_{\ell}, \text { open }_{\ell}\right)\right\} .
\end{aligned}
$$

We introduce shorthand notation for the above expression: $\pi \leftarrow \operatorname{NIPK}\left\{\left(\left(c_{1}: x_{1}\right), \ldots\right.\right.$, $\left.\left(c_{n}: x_{n}\right)\right)$ : Condition $\left.(x)\right\}$. For simplicity, we assume the proof $\pi$ includes $s$. 


\subsection{Groth-Sahai Commitments [GS07]}

We review the Groth-Sahai [GS07] commitment scheme. We use their scheme to commit to elements of a group $G$ of prime order $p$. Technically, their constructions commit to elements of certain modules, but we can apply them to certain bilinear groups elements. Groth and Sahai also have a construction for composite order groups using the Subgroup Decision assumption; however it lacks the necessary extraction properties.

$\operatorname{GSComSetup}(p, G, g)$. Outputs a common reference string params $_{C o m}$.

GSCommit $\left(\right.$ params $_{C o m}, x$, open). Takes as input $x \in G$ and some value open and outputs a commitment comm. The extension GSExpCommit (params Com $, b, \theta$, open) takes as input $\theta \in Z_{p}$ and a base $b \in G$ and outputs $(b, c o m m)$, where comm = GSCommit (params $\mathrm{Com}, b^{\theta}$, open). (Groth and Sahai compute commitments to elements in $Z_{p}$ slightly differently;

VerifyOpening $\left(\right.$ params $_{C o m}$, comm, $x$, open $)$. Takes $x \in G$ and open as input and outputs accept if comm is a commitment to $x$. To verify that $(b, c o m m)$ is a commitment to exponent $\theta$ check VerifyOpening $\left(\right.$ params $_{\mathrm{Com}}, \mathrm{comm}, b^{\theta}$, open $)$.

For brevity, we write GSCommit $(x)$ to indicate committing to $x \in G$ when the parameters are obvious and the value of open is chosen appropriately at random. Similarly, $\operatorname{GSExpCommit}(b, \theta)$ indicates committing to $\theta$ using $b \in G$ as the base.

GS commitments are perfectly binding, strongly computationally hiding, and extractable. Groth and Sahai [GS07] show how to instantiate commitments that meet these requirements using either the SXDH or DLIN assumptions. Commitments based on SXDH consist of 2 elements in $G$, while those based on DLIN setting require 3 elements in $G$. Note that in the Groth-Sahai proof system below, $G=G_{1}$ or $G=G_{2}$ for SXDH and $G=G_{1}=G_{2}$ for DLIN.

\subsection{Groth-Sahai Pairing Product Equation Proofs [GS07]}

Groth and Sahai [GS07] construct an $f$-extractable NIPK system that lets us prove statements in the context of groups with bilinear maps.

$\operatorname{GSSetup}\left(1^{k}\right)$ outputs $\left(p, G_{1}, G_{2}, G_{T}, e, g, h\right)$, where $G_{1}, G_{2}, G_{T}$ are groups of prime order $p$, with $g$ a generator of $G_{1}, h$ a generator of $G_{2}$, and $e: G_{1} \times G_{2} \rightarrow G_{T}$ a cryptographic bilinear map. GSSetup $\left(1^{k}\right)$ also outputs params $s_{1}$ and params $_{2}$ for constructing GS commitments in $G_{1}$ and $G_{2}$, respectively. (If the pairing is symmetric, $G_{1}=G_{2}$ and params $_{1}=$ params $_{2}$.) The statement $s$ to be proven consists of the following list of values: $\left\{a_{q}\right\}_{q=1 \ldots Q} \in G_{1},\left\{b_{q}\right\}_{q=1 \ldots Q} \in G_{2}, t \in G_{T}$, and $\left\{\alpha_{q, m}\right\}_{m=1 \ldots M, q=1 \ldots Q},\left\{\beta_{q, n}\right\}_{n=1 \ldots N, q=1 \ldots Q} \in Z_{p}$, as well as a list of commitments $\left\{c_{m}\right\}_{m=1 \ldots M}$ to values in $G_{1}$ and $\left\{d_{n}\right\}_{n=1 \ldots N}$ to values in $G_{2}$. Groth and Sahai show how to construct the following proof:

$$
\begin{aligned}
\operatorname{NIPK}\left\{\left(\left(c_{1}: x_{1}\right), \ldots,\left(c_{M}: x_{M}\right),\right.\right. & \left.\left(d_{1}: y_{1}\right), \ldots,\left(d_{N}: y_{N}\right)\right): \\
& \left.\prod_{q=1}^{Q} e\left(a_{q} \prod_{m=1}^{M} x_{m}^{\alpha_{q, m}}, b_{q} \prod_{n=1}^{N} y_{n}^{\beta_{q, n}}\right)=t\right\}
\end{aligned}
$$


The proof $\pi$ includes the statement being proven; this includes the commitments $c_{1}, \ldots$, $c_{M}$ and $d_{1}, \ldots, d_{N}$. Groth and Sahai provide an efficient extractor that opens these commitments to values $x_{1}, \ldots, x_{M}, y_{1}, \ldots, y_{N}$ that satisfy the pairing product equation.

Recall the function GSExpCommit params $_{1}, b, \theta$, open $)=(b$, GSCommit $\left(\right.$ params $_{1}, b^{\theta}$, open $\left.)\right)$. We can replace any of the clauses $\left(c_{m}: x_{m}\right)$ with the clause $\left(c_{m}: b^{\theta}\right)$, and add $b$ to the list of values included in the statement $s$ (and therefore in the proof $\pi$ ). The same holds for commitments $d_{n}$. Groth-Sahai proofs also allow us to prove that the openings of $\left(c_{1}, \ldots, c_{n}, d_{1}, \ldots, d_{n}\right)$ satisfy several equations simultaneously.

We formally define the Groth-Sahai proof system. Let $\operatorname{params}_{B M} \leftarrow \operatorname{BilinearSetup}\left(1^{k}\right)$.

GSSetup $\left(\right.$ params $\left._{B M}\right)$. Calls GSComSetup to generate params $_{1}$ and params $_{2}$ for constructing commitments in $G_{1}$ and $G_{2}$ respectively, and optional auxiliary values

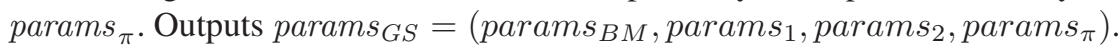

GSProve $\left(\right.$ params $_{G S}, s,\left(\left\{x_{m}\right\}_{1 \ldots M},\left\{y_{n}\right\}_{1 \ldots N}\right.$, openings $\left.)\right)$. Takes as input the parameters, the statement $s=\left\{\left(c_{1}, \ldots, c_{M}, d_{1}, \ldots, d_{N}\right)\right.$, equations $\}$ to be proven, (the statement $s$ includes the commitments and the parameters of the pairing product equations), the witness consisting of the values $\left\{x_{m}\right\}_{1 \ldots M},\left\{y_{n}\right\}_{1 \ldots N}$ and opening information openings. Outputs a proof $\pi$.

GSVerify $\left(\right.$ params $\left._{G S}, \pi\right)$. Returns accept if $\pi$ is valid, reject otherwise. (Note that it does not take the statement $s$ as input because we have assumed that the statement is always included in the proof $\pi$.)

GSExtractSetup $\left(\right.$ params $\left._{B M}\right)$. Outputs params $_{G S}$ and auxiliary information $\left(t d_{1}\right.$, $\left.t d_{2}\right)$. $\operatorname{params}_{G S}$ are distributed identically to the output of GSSetup $\left(\operatorname{params}_{B M}\right)$. $\left(t d_{1}, t d_{2}\right)$ allow an extractor to discover the contents of all commitments.

GSExtract $\left(\right.$ params $\left._{G S}, t d_{1}, t d_{2}, \pi\right)$. Outputs $x_{1}, \ldots, x_{M} \in G_{1}$ and $y_{1}, \ldots, y_{N} \in G_{2}$ that satisfy the equations and that correspond to the commitments (note that the commitments and the equations are included with the proof $\pi$ ).

Groth-Sahai proofs satisfy correctness, extractability, and strong witness indistinguishability. We explain these requirements in a manner compatible with our notation.

Correctness. An honest verifier always accepts a proof generated by an honest prover.

Extractability. If an honest verifier outputs accept, then the statement is true. This means that, given $t d_{1}, t d_{2}$ corresponding to params $_{G S}$, GSExtract extracts values from the commitments that satisfy the pairing product equations with probability 1.

Strong Witness Indistinguishability. A simulator Sim = (SimSetup, SimProve) with the following two properties exists: (1) SimSetup $\left(\operatorname{params}_{B M}\right)$ outputs params $_{G S}{ }^{\prime}$ such that they are computationally indistinguishable from the output of GSSetup( params $\left._{B M}\right)$. Let params $_{1}^{\prime} \in$ params $_{G S}{ }^{\prime}$ be the parameters for the commitment scheme in $G_{1}$. Using params $s_{1}^{\prime}$, commitments are perfectly hiding - this means that for all commitments comm, $\forall x \in G_{1}, \exists$ open : VerifyOpening $\left(\right.$ params $_{1}^{\prime}$, comm, $x$, open $)=$ accept (analogous for $G_{2}$ ). (2) Using the params ${ }_{G S}{ }^{\prime}$ generated by the challenger, GS proofs become perfectly witness indistinguishable. Suppose an unbounded adversary $\mathcal{A}$ generates a statement $s$ consisting of the pairing product equations and a set of commitments $\left(c_{1}, \ldots, c_{M}, d_{1}, \ldots, d_{N}\right)$. The adversary opens 
the commitments in two different ways $W_{0}=\left(x_{1}^{(0)}, \ldots, x_{M}^{(0)}, y_{1}^{(0)}, \ldots, y_{N}^{(0)}\right.$, openings $\left._{0}\right)$ and $W_{1}=\left(x_{1}^{(1)}, \ldots, x_{M}^{(1)}, y_{1}^{(1)}, \ldots, y_{N}^{(1)}\right.$, openings $\left.{ }_{1}\right)$ (under the requirement that these witnesses must both satisfy $s$ ). The values opening $s_{b}$ show how to open the commitments to $\left\{x_{m}^{(b)}, y_{n}^{(b)}\right\}$. (The adversary can do this because it is unbounded.) The challenger gets the statement $s$ and the two witnesses $W_{0}$ and $W_{1}$. He chooses a bit $b \leftarrow\{0,1\}$ and computes $\pi=$ GSProve $\left(\right.$ params $\left._{G S}{ }^{\prime}, s, W_{b}\right)$. Strong witness indistinguishability means that $\pi$ is distributed independently of $b$.

Composable Zero-Knowledge. Note that Groth and Sahai show that if in a given pairing product equation the constant $t$ can be written as $t=e\left(t_{1}, t_{2}\right)$ for known $t_{1}, t_{2}$, then these proofs can be done in zero knowledge. However, their zero knowldge proof construction is significantly less efficient than the WI proofs. Thus, we choose to use only the WI construction as a building block. Then we can take advantage of special features of our P-signature construction to create much more efficient proofs that still have the desired zero knowledge properties. The only exception is our construction for EqCommProve, which does use the zero knowledge technique suggested by Groth and Sahai.

\subsection{Proofs About Committed Exponents}

We use the Groth-Sahai proof system to prove equality of committed exponents.

Equality of Committed Exponents in Different Groups. We want to prove the statement NIPK $\left\{\left(\left(c: g^{\alpha}\right),\left(d: h^{\beta}\right)\right): \alpha=\beta\right\}$. We perform a Groth-Sahai pairing product equation proof $\operatorname{NIPK}\{((c: x),(d: y)): e(x, h) e(1 / g, y)=1\}$. Security is straightforward due to the $f$-extractability property of the GS proof system.

Equality of Committed Exponents in the Same Group. We want to prove the statement NIPK $\left\{\left(\left(c_{1}: g^{\alpha}\right),\left(c_{2}: u^{\beta}\right)\right): \alpha=\beta\right\}$, where $g, u \in G_{1}$. This is equivalent to proving NIPK $\left\{\left(\left(c_{1}: g^{\alpha}\right),\left(c_{2}: u^{\beta}\right),\left(d: h^{\gamma}\right): \alpha=\gamma \wedge \beta=\gamma\right\}\right.$.

Zero-Knowledge Proof of Equality of Committed Exponents. We want to prove the statement NIZKPK $\left\{\left(\left(c_{1}: g^{\alpha}\right),\left(c_{2}: g^{\beta}\right): \alpha=\beta\right\}\right.$ in zero-knowledge. We perform the Groth-Sahai zero-knowledge pairing product equation proof NIPK $\left\{\left(\left(c_{1}: g^{\alpha}\right),\left(c_{2}\right.\right.\right.$ : $\left.\left.g^{\beta}\right),\left(d: h^{\theta}\right): e\left(a / b, h^{\theta}\right)=1 \wedge e\left(g, h^{\theta}\right) e(1 / g, h)=1\right\}$. Proof of equality of committed exponents in group $G_{2}$ is done analogously. See full version for details.

Remark 1. We cannot directly use Groth-Sahai general arithmetic gates [GS07] to construct the above proofs because they assume that the commitments use the same base.

\section{Efficient Construction of P-signature Scheme}

In this section, we present a new signature scheme and then build a P-signature scheme from it. The new signature scheme is inspired by the full Boneh-Boyen signature scheme, and is as follows:

New-SigSetup $\left(1^{k}\right)$ runs BilinearSetup $\left(1^{k}\right)$ to get the pairing parameters $\left(p, G_{1}, G_{2}, G_{T}, e, g, h\right)$. In the sequel, by $z$ we denote $z=e(g, h)$. 
New-Keygen (params) picks a random $\alpha, \beta \leftarrow Z_{p}$. The signer calculates $v=h^{\alpha}$, $w=h^{\beta}, \tilde{v}=g^{\alpha}, \tilde{w}=g^{\beta}$. The secret-key is $s k=(\alpha, \beta)$. The public-key is $p k=(v, w, \tilde{v}, \tilde{w})$. The public key can be verified by checking that $e(g, v)=e(\tilde{v}, h)$ and $e(g, w)=e(\tilde{w}, h)$.

New-Sign $($ params, $(\alpha, \beta), m)$ chooses $r \leftarrow Z_{p}-\left\{\frac{\alpha-m}{\beta}\right\}$ and calculates $C_{1}=$ $g^{1 /(\alpha+m+\beta r)}, C_{2}=w^{r}, C_{3}=u^{r}$. The signature is $\left(C_{1}, C_{2}, C_{3}\right)$.

New-VerifySig $\left(\right.$ params, $\left.(v, w, \tilde{v}, \tilde{w}), m,\left(C_{1}, C_{2}, C_{3}\right)\right) \quad$ outputs $\quad$ accept $\quad$ if $e\left(C_{1}, v h^{m} C_{2}\right)=z, e\left(u, C_{2}\right)=e\left(C_{3}, w\right)$, and if the public key is correctly formed, i.e., $e(g, v)=e(\tilde{v}, h)$, and $e(g, w)=e(\tilde{w}, h) !$

Theorem 1. Let $F(x)=\left(h^{x}, u^{x}\right)$, where $u \in G_{1}$ and $h \in G_{2}$ as in the HSDH and TDH assumptions. Our new signature scheme is F-secure given HSDH and TDH. (See full version for proof.)

We extend the above signature scheme to obtain our second P-signature scheme (Setup, Keygen, Sign, VerifySig, Commit, ObtainSig, IssueSig, Prove, VerifyProof, EqCommProve, VerEqComm). The algorithms are as follows:

Setup $\left(1^{k}\right)$ First, obtain params $s_{B M}=\left(p, G_{1}, G_{2}, G_{T}, e, g, h\right) \leftarrow$ BilinearSetup $\left(1^{k}\right)$. Next, obtain params paS $_{\text {params }}=\left(\right.$ par $_{B}$, params $_{1}$, params $_{2}$, params $\left._{\pi}\right) \leftarrow$ GSSetup $\left(\right.$ params $\left._{B M}\right)$. Pick $u \leftarrow G_{1}$. Let params $=\left(\right.$ params $\left._{G S}, u\right)$. As before, $z$ is defined as $z=e(g, h)$.

Keygen (params) Run the New-Keygen $\left(\right.$ params $\left._{B M}\right)$ and output $s k=(\alpha, \beta), p k=$ $\left(h^{\alpha}, h^{\beta}, g^{\alpha}, g^{\beta}\right)=(v, w, \tilde{v}, \tilde{w})$.

Sign (params, sk, $m)$ Run New-Sign $\left(\right.$ params $\left._{B M}, s k, m\right)$ to obtain $\sigma=\left(C_{1}, C_{2}, C_{3}\right)$ where $C_{1}=g^{1 /(\alpha+m+\beta r)}, C_{2}=w^{r}, C_{3}=u^{r}$, and $s k=(\alpha, \beta)$

VerifySig (params, $p k, m, \sigma)$ Run New-VerifySig $\left(\right.$ params $\left._{B M}, p k, m, \sigma\right)$.

Commit(params, $m$, open) To commit to $m$, compute $C=$ GSExpCommit $\left(\right.$ params $_{2}, h, m$, open). (Recall that GSExpCommit $\left(\right.$ params $_{2}, h$, $m$,open $)=$ GSCommit $\left(\right.$ params $_{2}, h^{m}$, open $)$, and params $_{2}$ is part of params $_{G S}$.)

ObtainSig (params, pk, $m$, comm, open $) \leftrightarrow$ IssueSig(params, sk, comm). The user and the issuer run the following protocol:

1. The user chooses $\rho_{1}, \rho_{2} \leftarrow Z_{p}$.

2. The issuer chooses $r^{\prime} \leftarrow Z_{p}$.

3. The user and the issuer run a secure two-party computation protocol where the user's private inputs are $\left(\rho_{1}, \rho_{2}, m\right.$, open $)$, and the issuer's private inputs are $s k=(\alpha, \beta)$ and $r^{\prime}$.

The issuer's private output is $x=\left(\alpha+m+\beta \rho_{1} r^{\prime}\right) \rho_{2}$ if comm $=$ Commit (params, $m$, open), and $x=\perp$ otherwise.

4. If $x \neq \perp$, the issuer calculates $C_{1}^{\prime}=g^{1 / x}, C_{2}^{\prime}=w^{r^{\prime}}$ and $C_{3}^{\prime}=u^{r^{\prime}}$, and sends $\left(C_{1}^{\prime}, C_{2}^{\prime}, C_{3}^{\prime}\right)$ to the user.

5. The user computes $C_{1}=C_{1}^{\prime \rho_{2}}, C_{2}=C_{2}^{\prime \rho_{1}}$, and $C_{3}=C_{3}^{\prime \rho_{1}}$ and then verifies that the signature $\left(C_{1}, C_{2}, C_{3}\right)$ is valid.

\footnotetext{
${ }^{1}$ The latter is needed only once per public key, and is meaningless in a symmetric pairing setting.
} 
Prove $($ params, $p k, m, \sigma)$ Check if $p k$ and $\sigma$ are valid, and if they are not, output

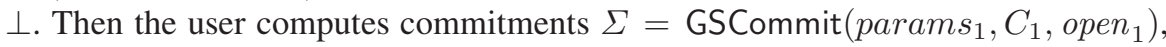

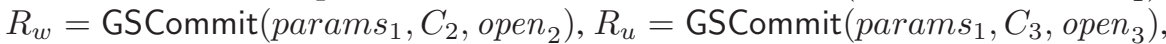
$M_{h}=$ GSExpCommit $\left(\right.$ params $_{2}, h$, m, open 4$)=$ GSCommit $\left(\right.$ param $_{2}, h^{m}$, open $\left._{4}\right)$ and $M_{u}=$ GSExpCommit $\left(\right.$ params $_{1}, u, m$, open $\left._{5}\right)=$ GSCommit $\left(\right.$ params $_{1}, u^{m}$, open $\left._{5}\right)$.

The user outputs the commitment comm $=M_{h}$ and the proof

$$
\begin{aligned}
\pi=\operatorname{NIPK}\left\{\left(\left(\Sigma: C_{1}\right),\left(R_{w}: C_{2}\right),\left(R_{u}: C_{3}\right)\left(M_{h}: h^{\alpha}\right),\left(M_{u}: u^{\beta}\right)\right):\right. \\
\left.e\left(C_{1}, v h^{\alpha} C_{2}\right)=z \wedge e\left(u, C_{2}\right)=e\left(C_{3}, w\right) \wedge \alpha=\beta\right\} .
\end{aligned}
$$

VerifyProof (params, $p k, c o m m, \pi$ ) Outputs accept if the proof $\pi$ is a valid proof of the statement described above for $M_{h}=c o m m$ and for properly formed $p k$.

EqCommProve (params, $m$, open, open') Let commitment comm = Commit $($ params,$m$, open $)=$ GSCommit $\left(\right.$ params $_{2}, h^{m}$,open $)$ and comm ${ }^{\prime}=$ Commit(params, $m$, open $\left.{ }^{\prime}\right)=$ GSCommit $\left(\right.$ params $_{2}, h^{m}$, open $\left.{ }^{\prime}\right)$. Use the GS proof system as described in Section 4.4 to compute $\pi \leftarrow \operatorname{NIZKPK}\{(($ comm : $\left.h^{\alpha}\right),\left(\right.$ comm $\left.\left.^{\prime}: h^{\beta}\right): \alpha=\beta\right\}$.

VerEqComm (params, comm, comm $\left.{ }^{\prime}, \pi\right)$ Verify the proof $\pi$ using the GS proof system as described in Section 4.4

Theorem 2 (Efficiency). Using SXDH GS proofs, each P-signature proof for our new signature scheme consists of 18 elements in $G_{1}$ and 16 elements in $G_{2}$. The prover performs 34 multi-exponentiation and the verifier 68 pairings. Using DLIN, each $P$ signature proof consists of 42 elements in $G_{1}=G_{2}$. The prover has to do 42 multiexponentiations and the verifier 84 pairings.

Theorem 3 (Security). Our second P-signature construction is secure given HSDH and TDH and the security of the GS commitments and proofs.

Proof. Correctness. VerifyProof will always accept properly formed proofs.

Signer Privacy. We must construct the Simlssue algorithm that is given as input params, a commitment comm and a signature $\sigma=\left(C_{1}, C_{2}, C_{3}\right)$ and must simulate the adversary's view. Simlssue will invoke the simulator for the two-party computation protocol. Recall that in two-party computation, the simulator can first extract the input of the adversary: in this case, some $\left(\rho_{1}, \rho_{2}, m\right.$, open $)$. Then Simlssue checks that comm $=$ Commit(params, $m$, open $)$; if it isn't, it terminates. Otherwise, it sends to the adversary the values $\left(C_{1}^{\prime}=C_{1}^{1 / \rho_{2}}, C_{2}^{\prime}=C_{2}^{1 / \rho_{1}}, C_{3}^{\prime}=C_{3}^{1 / \rho_{1}}\right)$. Suppose the adversary can determine that it is talking with a simulator. Then it must be the case that the adversary's input to the protocol was incorrect which breaks the security properties of the two-party computation.

User Privacy. The simulator will invoke the simulator for the two-party computation protocol. Recall that in two-party computation, the simulator can first extract the input of the adversary (in this case, some $\left(\alpha^{\prime}, \beta^{\prime}\right)$, not necessarily the valid secret key). Then the simulator is given the target output of the computation (in this case, the value $x$ 
which is just a random value that the simulator can pick itself), and proceeds to interact with the adversary such that if the adversary completes the protocol, its output is $x$. Suppose the adversary can determine that it is talking with a simulator. Then it breaks the security of the two-party computation protocol.

Zero knowledge. Consider the following algorithms. SimSetup runs BilinearSetup to get params $_{B M}=\left(p, G_{1}, G_{2}, G_{T}, e, g, h\right)$. It then picks $t \leftarrow Z_{p}$ and sets up $u=$ $g^{a}$. Next it calls GSSimSetup $\left(\right.$ params $\left._{B M}\right)$ to obtain params $_{G S}$ and sim. The final parameters are params $=\left(\right.$ params $\left._{G S}, u, z=e(g, h)\right)$ and $\operatorname{sim}=(a$, sim $)$. Note that the distribution of params is indistinguishable from the distribution output by Setup. SimProve receives params, sim, and public key $(v, \tilde{v}, w, \tilde{w})$ and can use trapdoor sim to create a random P-signature forgery in SimProve as follows. Pick $s, r \leftarrow Z_{p}$ and compute $\sigma=g^{1 / s}$. We implicitly set $m=s-\alpha-r \beta$. Note that the simulator does not know $m$ and $\alpha$. However, he can compute $h^{m}=h^{s} /\left(v w^{r}\right)$ and $u^{m}=u^{s} /\left(\tilde{v}^{a} \tilde{w}^{a r}\right)$. Now he can use $\sigma, h^{m}, u^{m}, w^{r}, u^{r}$ as a witness and construct the proof $\pi$ in the same way as the real Prove protocol. By the witness indistinguishability of the GS proof system, a proof using the faked witnesses is indistinguishable from a proof using a real witness, thus SimProve is indistinguishable from Prove.

Finally, we need to show that we can simulate proofs of EqCommProve given the trapdoor $\operatorname{sim}_{G S}$. This follows directly from composable zero knowledge of EqCommProve. See full version for details.

Unforgeability. Consider the following algorithms: ExtractSetup $\left(1^{k}\right)$ outputs the usual params, except that it invokes GSExtractSetup to get alternative params $_{G S}$ and the trapdoor $t d=\left(t d_{1}, t d_{2}\right)$ for extracting GS commitments in $G_{1}$ and $G_{2}$. The parameters generated by GSSetup are indistinguishable from those generated by

GSExtractSetup, so we know that the parameters generated by ExtractSetup will be indistinguishable from those genrated by Setup.

Extract (params, $t d, c o m m, \pi$ ) extracts the values from commitment comm and the commitments $M_{h}, M_{u}$ contained in the proof $\pi$ using the GS commitment extractor. If VerifyProof accepts then comm $=M_{h}$. Let $F(m)=\left(h^{m}, u^{m}\right)$.

Now suppose we have an adversary that can break the unforgeability of our Psignature scheme for this extractor and this bijection.

A P-signature forger outputs a proof from which we extract $(F(m), \sigma)$ such that either (1) VerifySig(params, $p k, m, \sigma)=$ reject, or (2) comm is not a commitment to $m$, or (3) the adversary never queried us on $m$. Since VerifyProof checks a set of pairing product equations, $f$-extractability of the GS proof system trivially ensures that (1) never happens. Since VerifyProof checks that $M_{h}=c o m m$, this ensures that (2) never happens. Therefore, we consider the third possibility. The extractor calcualtes $F(m)=\left(h^{m}, u^{m}\right)$ where $m$ is fresh. Due to the randomness element $r$ in the signature scheme, we have two types of forgeries. In a Type 1 forgery, the extractor can extract from the proof a tuple of the form $\left(g^{1 /(\alpha+m+\beta r)}, w^{r}, u^{r}, h^{m}, u^{m}\right)$, where $m+r \beta \neq$ $m_{\ell}+r_{\ell} \beta$ for any $\left(m_{\ell}, r_{\ell}\right)$ used in answering the adversary's signing or proof queries. The second type of forgery is one where $m+r \beta=m_{\ell}+r_{\ell} \beta$ for $\left(m_{\ell}, r_{\ell}\right)$ used in one of these previous queries. We show that a Type 1 forger can be used to break the HSDH assumption, and a Type 2 forger can be used to break the TDH assumption. 
Type 1 forgeries: $\beta r+m \neq \beta r_{\ell}+m_{\ell}$ for any $r_{\ell}, m_{\ell}$ from a previous query. The reduction gets an instance of the $\mathrm{HSDH}$ problem $\left(p, G_{1}, G_{2}, G_{T}, e, g, X, \tilde{X}, h, u\right.$, $\left.\left\{C_{\ell}, H_{\ell}, U_{\ell}\right\}_{\ell=1 \ldots q}\right)$, such that $X=h^{x}$ and $\tilde{X}=g^{x}$ for some unknown $x$, and for all $\ell$, $C_{\ell}=g^{1 /\left(x+c_{\ell}\right)}, H_{\ell}=h^{c_{\ell}}$, and $U_{\ell}=u^{c_{\ell}}$ for some unknown $c_{\ell}$. The reduction sets up the parameters of the new signature scheme as $\left(p, G_{1}, G_{2}, e, g, h, u, z=e(g, h)\right)$. Next, the reduction chooses $\beta \leftarrow Z_{p}$, sets $v=X, \tilde{v}=\tilde{X}$ and calculates $w=h^{\beta}, \tilde{w}=g^{\beta}$. The reduction gives the adversary the public parameters, the trapdoor, and the publickey $(v, w, \tilde{v}, \tilde{w})$.

Suppose the adversary's $\ell$ th query is to Sign message $m_{\ell}$. The reduction will implicitly set $r_{\ell}$ to be such that $c_{\ell}=m_{\ell}+\beta r_{\ell}$. This is an equation with two unknowns, so we do not know $r_{\ell}$ and $c_{\ell}$. The reduction sets $C_{1}=C_{\ell}$. It computes $C_{2}=H_{\ell} / h^{m_{\ell}}=h^{c_{\ell}} / h^{m_{\ell}}=w^{r_{\ell}}$. Then it computes $C_{3}=\left(U_{\ell}\right)^{1 / \beta} / u^{m_{\ell} / \beta}=$ $\left(u^{c_{\ell}}\right)^{1 / \beta} / u^{m_{\ell} / \beta}=u^{\left(c_{\ell}-m_{\ell}\right) / \beta}=u^{r_{\ell}}$ The reduction returns the signature $\left(C_{1}, C_{2}, C_{3}\right)$.

Eventually, the adversary returns a proof $\pi$. Since $\pi$ is $f$-extractable and perfectly sound, we extract $\sigma=g^{1 /(x+m+\beta r)}, a=w^{r}, b=u^{r}, c=h^{m}$, and $d=u^{m}$. Since this is a P-signature forgery, $(c, d)=\left(h^{m}, u^{m}\right) \notin F\left(Q_{\text {Sign }}\right)$. Since this is a Type 1 forger, we also have that $m+\beta r \neq m_{\ell}+\beta r_{\ell}$ for any of the adversary's previous queries. Therefore, $\left.\left(\sigma, c a, d b^{\beta}\right)=\left(g^{1 /(x+m+\beta r}\right), h^{m+\beta r}, u^{m+\beta r}\right)$ is a new HSDH tuple.

Type 2 forgeries: $\beta r+m=\beta r_{\ell}+m_{\ell}$ for some $r_{\ell}, m_{\ell}$ from a previous query. The reduction receives $\left(p, G_{1}, G_{2}, G_{T}, e, g, h, X, Z, Y,\left\{\sigma_{\ell}, c_{\ell}\right\}\right)$, where $X=h^{x}, Z=g^{x}$, $Y=g^{y}$, and for all $\ell, \sigma_{\ell}=g^{1 /\left(x+c_{\ell}\right)}$. The reduction chooses $\gamma \leftarrow Z_{p}$ and sets $u=Y^{\gamma}$. The reduction sets up the parameters of the new signature scheme as $\left(p, G_{1}, G_{2}, e, g, h, u, z=e(g, h)\right)$. Next the reduction chooses $\alpha \leftarrow Z_{p}$, and calculates $v=h^{\alpha}, w=X^{\gamma}, \tilde{v}=g^{\alpha}, \tilde{w}=Z^{\gamma}$. It gives the adversary the parameters, the trapdoor, and the public-key $(v, w, \tilde{v}, \tilde{w})$. Note that we set up our parameters and public-key so that $\beta$ is implicitly defined as $\beta=x \gamma$, and $u=g^{\gamma y}$.

Suppose the adversary's $\ell$ th query is to Sign message $m_{\ell}$. The reduction sets $r_{\ell}=$ $\left(\alpha+m_{\ell}\right) /\left(c_{\ell} \gamma\right)$ (which it can compute). The reduction computes $C_{1}=\sigma_{\ell}^{1 /\left(\gamma r_{\ell}\right)}=$ $\left(g^{1 /\left(x+c_{\ell}\right)}\right)^{1 /\left(\gamma r_{\ell}\right)}=g^{1 /\left(\gamma r_{\ell}\left(x+c_{\ell}\right)\right)}=g^{1 /\left(\alpha+m_{\ell}+\beta r_{\ell}\right)}$. Since the reduction knows $r_{\ell}$, it computes $C_{2}=w^{r_{\ell}}, C_{3}=u^{r_{\ell}}$ and send $\left(C_{1}, C_{2}, C_{3}\right)$ to $\mathcal{A}$.

Eventually, the adversary returns a proof $\pi$. The proof $\pi$ is $f$-extractable and perficetly sound, the reduction can extract $\sigma=g^{1 /(x+m+\beta r)}, a=w^{r}, b=u^{r}, c=h^{m}$, and $d=u^{m}$. Therefore, VerifySig will always accept $m=F^{-1}(c, d), \sigma, a, b$. We also know that if this is a forgery, then VerifyProof accepts, which means that $\operatorname{com} m=M_{h}$, which is a commitment to $m$. Thus, since this is a P-signature forgery, it must be the case that $(c, d)=\left(h^{m}, u^{m}\right) \notin F\left(Q_{\text {Sign }}\right)$. However, since this is a Type 2 forger, we also have that $\exists \ell: m+\beta r=m_{\ell}+\beta r_{\ell}$, where $m_{\ell}$ is one of the adversary's previous Sign or Prove queries. We implicitly define $\delta=m-m_{\ell}$. Since $m+\beta r=m_{\ell}+\beta r_{\ell}$, we also get that $\delta=\beta\left(r_{\ell}-r\right)$. Using $\beta=x \gamma$, we get that $\delta=x \gamma\left(r_{\ell}-r\right)$. We compute: $A=c / h^{m_{\ell}}=h^{m-m_{\ell}}=h^{\delta}, B=u^{r_{\ell}} / b=u^{r_{\ell}-r}=u^{\delta /(\gamma x)}=g^{y \delta / x}$ and $C=\left(d / u^{m_{\ell}}\right)^{1 / \gamma}=u^{\left(m-m_{\ell}\right) / \gamma}=u^{\delta / \gamma}=g^{\delta y}$. We implicitly set $\mu=\delta / x$, thus $(A, B, C)=\left(h^{\mu x}, g^{\mu y}, g^{\mu x y}\right)$ is a valid TDH tuple.

Acknowledgments. Mira Belenkiy, Melissa Chase and Anna Lysyanskaya are supported by NSF grants CNS-0374661 CNS-0627553. Markulf Kohlweiss is supported 
by the European Commission's IST Program under Contracts IST-2002-507591 PRIME and IST-2002-507932 ECRYPT.

\section{References}

[ACJT00] Ateniese, G., Camenisch, J., Joye, M., Tsudik, G.: A practical and provably secure coalition-resistant group signature scheme. In: Bellare, M. (ed.) CRYPTO 2000. LNCS, vol. 1880, pp. 255-270. Springer, Heidelberg (2000)

[BB04] Boneh, D., Boyen, X.: Short signatures without random oracles. In: Cachin, C., Camenisch, J.L. (eds.) EUROCRYPT 2004. LNCS, vol. 3027, pp. 54-73. Springer, Heidelberg (2004)

[BBS04] Boneh, D., Boyen, X., Shacham, H.: Short group signatures using strong DiffieHellman. In: Franklin, M. (ed.) CRYPTO 2004. LNCS, vol. 3152, pp. 41-55. Springer, Heidelberg (2004)

[BCC04] Brickell, E., Camenisch, J., Chen, L.: Direct anonymous attestation. Technical Report Research Report RZ 3450, IBM Research Division (March 2004)

[BCL04] Bangerter, E., Camenisch, J., Lysyanskaya, A.: A cryptographic framework for the controlled release of certified data. In: Cambridge Security Protocols Workshop (2004)

[BDMP91] Blum, M., De Santis, A., Micali, S., Persiano, G.: Non-interactive zero-knowledge. SIAM J. of Computing 20(6), 1084-1118 (1991)

[BFM88] Blum, M., Feldman, P., Micali, S.: Non-interactive zero-knowledge and its applications (extended abstract). In: STOC 1988, pp. 103-112 (1988)

[Bra93] Brands, S.: An efficient off-line electronic cash system based on the representation problem. Technical Report CS-R9323, CWI (April 1993)

[Bra99] Brands, S.: Rethinking Public Key Infrastructure and Digital Certificates- Building in Privacy. PhD thesis, Eindhoven Inst. of Tech. The Netherlands (1999)

[BW06] Boyen, X., Waters, B.: Compact group signatures without random oracles. In: Vaudenay, S. (ed.) EUROCRYPT 2006. LNCS, vol. 4004, pp. 427-444. Springer, Heidelberg (2006)

[BW07] Boyen, X., Waters, B.: Full-domain subgroup hiding and constant-size group signatures. In: Okamoto, T., Wang, X. (eds.) PKC 2007. LNCS, vol. 4450, pp. 1-15. Springer, Heidelberg (2007)

[CFN90] Chaum, D., Fiat, A., Naor, M.: Untraceable electronic cash. In: Menezes, A., Vanstone, S.A. (eds.) CRYPTO 1990. LNCS, vol. 537, pp. 319-327. Springer, Heidelberg (1991)

[CGH04] Canetti, R., Goldreich, O., Halevi, S.: The random oracle methodology, revisited. J. ACM 51(4), 557-594 (2004)

[Cha85] Chaum, D.: Security without identification: Transaction systems to make big brother obsolete. Communications of the ACM 28(10), 1030-1044 (1985)

$\left[\mathrm{CHK}^{+}\right.$06] Camenisch, J., Hohenberger, S., Kohlweiss, M., Lysyanskaya, A., Meyerovich, M.: How to win the clonewars: efficient periodic n-times anonymous authentication. In: CCS 2006, pp. 201-210 (2006)

[CHL05] Camenisch, J., Hohenberger, S., Lysyanskaya, A.: Compact E-Cash. In: Cramer, R.J.F. (ed.) EUROCRYPT 2005. LNCS, vol. 3494, pp. 302-321. Springer, Heidelberg (2005)

[CL01] Camenisch, J., Lysyanskaya, A.: Efficient non-transferable anonymous multi-show credential system with optional anonymity revocation. In: Pfitzmann, B. (ed.) EUROCRYPT 2001. LNCS, vol. 2045, pp. 93-118. Springer, Heidelberg (2001) 
[CL02] Camenisch, J., Lysyanskaya, A.: A signature scheme with efficient protocols. In: Cimato, S., Galdi, C., Persiano, G. (eds.) SCN 2002. LNCS, vol. 2576, pp. 268289. Springer, Heidelberg (2003)

[CL04] Camenisch, J., Lysyanskaya, A.: Signature schemes and anonymous credentials from bilinear maps. In: Franklin, M. (ed.) CRYPTO 2004. LNCS, vol. 3152, pp. 56-72. Springer, Heidelberg (2004)

[CLM07] Camenisch, J., Lysyanskaya, A., Meyerovich, M.: Endorsed e-cash. In: IEEE Symposium on Security and Privacy 2007, pp. 101-115 (2007)

[CP93] Chaum, D., Pedersen, T.: Transferred cash grows in size. In: Rueppel, R.A. (ed.) EUROCRYPT 1992. LNCS, vol. 658, pp. 390-407. Springer, Heidelberg (1993)

[CS97] Camenisch, J., Stadler, M.: Efficient group signature schemes for large groups. In: Kaliski Jr., B.S. (ed.) CRYPTO 1997. LNCS, vol. 1294, pp. 410-424. Springer, Heidelberg (1997)

[CvH91] Chaum, D., van Heyst, E.: Group signatures. In: Davies, D.W. (ed.) EUROCRYPT 1991. LNCS, vol. 547, pp. 257-265. Springer, Heidelberg (1991)

[CVH02] Camenisch, J., Van Herreweghen, E.: Design and implementation of the idemix anonymous credential system. In: Proc. 9th ACM CCS 2002, pp. 21-30 (2002)

[Dam90] Damgård, I.: Payment systems and credential mechanism with provable security against abuse by individuals. In: Goldwasser, S. (ed.) CRYPTO 1988. LNCS, vol. 403, pp. 328-335. Springer, Heidelberg (1990)

[DDP00] De Santis, A., Di Crescenzo, G., Persiano, G.: Necessary and sufficient assumptions for non-interactive zero-knowledge proofs of knowledge for all NP relations. In: ICALP 2000, pp. 451-462 (2000)

[DDP06] Damgård, I., Dupont, K., Pedersen, M.: Unclonable group identification. In: Vaudenay, S. (ed.) EUROCRYPT 2006. LNCS, vol. 4004, pp. 555-572. Springer, Heidelberg (2006)

[DNRS03] Dwork, C., Naor, M., Reingold, O., Stockmeyer, L.J.: Magic functions. J. ACM 50(6), 852-921 (2003)

[DSMP88] De Santis, A., Micali, S., Persiano, G.: Non-interactive zero-knowledge proof systems. In: Pomerance, C. (ed.) CRYPTO 1987. LNCS, vol. 293, pp. 52-72. Springer, Heidelberg (1988)

[FO98] Fujisaki, E., Okamoto, T.: A practical and provably secure scheme for publicly verifiable secret sharing and its applications. In: Nyberg, K. (ed.) EUROCRYPT 1998. LNCS, vol. 1403, pp. 32-46. Springer, Heidelberg (1998)

[FS87] Fiat, A., Shamir, A.: How to prove yourself: Practical solutions to identification and signature problems. In: Odlyzko, A.M. (ed.) CRYPTO 1986. LNCS, vol. 263, pp. 186-194. Springer, Heidelberg (1987)

[GK03] Goldwasser, S., Kalai, Y.: On the (in)security of the Fiat-Shamir paradigm. In: FOCS 2003, pp. 102-115 (2003)

[GMR88] Goldwasser, S., Micali, S., Rivest, R.: A digital signature scheme secure against adaptive chosen-message attacks. SIAM J. on Computing 17(2), 281-308 (1988)

[GMW86] Goldreich, O., Micali, S., Wigderson, A.: Proofs that yield nothing but their validity and a method of cryptographic protocol design. In: FOCS 1986, pp. 174-187 (1986)

[GS07] Groth, J., Sahai, A.: Efficient non-interactive proof systems for bilinear groups, http: //eprint.iacr.org/2007/155

[JS04] Jarecki, S., Shmatikov, V.: Handcuffing big brother: an abuse-resilient transaction escrow scheme. In: Cachin, C., Camenisch, J.L. (eds.) EUROCRYPT 2004. LNCS, vol. 3027, pp. 590-608. Springer, Heidelberg (2004)

[LRSW99] Lysyanskaya, A., Rivest, R., Sahai, A., Wolf, S.: Pseudonym systems. In: Emmerich, W., Tai, S. (eds.) EDO 2000. LNCS, vol. 1999, Springer, Heidelberg (2001) 
[Lys02] Lysyanskaya, A.: Signature Schemes and Applications to Cryptographic Protocol Design. PhD thesis, MIT, Cambridge, Massachusetts (September 2002)

[Nao03] Naor, M.: On cryptographic assumptions and challenges. In: Boneh, D. (ed.) CRYPTO 2003. LNCS, vol. 2729, pp. 96-109. Springer, Heidelberg (2003)

[Ped92] Pedersen, T.: Non-interactive and information-theoretic secure verifiable secret sharing. In: Brickell, E.F. (ed.) CRYPTO 1992. LNCS, vol. 740, pp. 129-140. Springer, Heidelberg (1993)

[Sco02] Scott, M.: Authenticated id-based key exchange and remote log-in with insecure token and pin number, http://eprint.iacr.org/2002/164

[TFS04] Teranishi, I., Furukawa, J., Sako, K.: $k$-times anonymous authentication (extended abstract). In: Lee, P.J. (ed.) ASIACRYPT 2004. LNCS, vol. 3329, pp. 308-322. Springer, Heidelberg (2004)

[TS06] Teranishi, I., Sako, K.: $k$-times anonymous authentication with a constant proving cost. In: Yung, M., Dodis, Y., Kiayias, A., Malkin, T.G. (eds.) PKC 2006. LNCS, vol. 3958, pp. 525-542. Springer, Heidelberg (2006)

[Yao86] Yao, A.: How to generate and exchange secrets. In: FOCS 1986, pp. 162-167 (1986) 